\title{
Indígenas en contextos urbanos. Cañaris, otavaleños y saraguros en la ciudad de Cuenca (Ecuador)
}

Indigenous people in urban contexts: Canaris, Otavaleños and Saraguros in the city of Cuenca (Ecuador)

José María Valcuende del Río

Universidad Pablo de Olavide (España). Investigador invitado de la Universidad de Cuenca (Ecuador). Proyecto Prometeo (SENECYT).

jmvalrio@upo.es

\author{
Piedad Vásquez Andrade \\ Aula de Derechos Humanos. Universidad de Cuenca. Ecuador \\ piedadva66@hotmail.com \\ Fredy Javier Hurtado Landy \\ Aula de Derechos Humanos. Universidad de Cuenca. Ecuador \\ frederick_land@yahoo.es
}

\section{MINORÍAS ÉTNICAS, PROCESOS DE GLOBALIZACIÓN Y CONTEXTOS URBANOS \\ MONOGRÁFICO COORDINADO POR JOSÉ Ma VALCUENDE (Univ. Pablo de Olavide) e ISRAEL S. IDROVO LANDY (Univ. de Cuenca, Ecuador)}

\section{RESUMEN}

Las ciudades coloniales en América Latina tienden a ocultar la presencia de las poblaciones indígenas actuales a partir de su anclaje discursivo a un pasado imaginario. En este artículo nos planteamos aproximarnos a la situación de tres poblaciones indígenas: cañaris, saraguros y otavaleños en la ciudad de Cuenca (Ecuador), con el fin de analizar los elementos que favorecen o dificultan sus estrategias de visibilización o invisibilización en el contexto urbano.

\footnotetext{
ABSTRACT these indigenous communities in urban contexts.

PALABRAS CLAVE

indígenas urbanos | cañaris | otavaleños | saraguros | Cuenca

KEYWORDS

indigenous people | Cañaris | Otavaleños | Saraguros | Cuenca
}

Latin American colonial cities quite often try to conceal the current presence and existence of indigenous communities by placing them in a discourse of a remote and imaginary past that has no connection with the present. In this paper, we study this phenomenon with regard to three different indigenous groups (Cañaris, Saraguros, and Otavaleños) in the city of Cuenca (Ecuador). Our analysis focuses mainly on the instruments and strategies that seek either the visualization or concealment of

\section{Introducción}

Discursivamente, "lo indígena" y "lo urbano" se presentan como realidades incompatibles desde una doble perspectiva que los naturaliza o muestra como mera reminiscencia del pasado, produciéndose un doble alejamiento: 1) espacial, al representarlos como parte indisociable de un mundo no urbano; y 2) temporal, al reivindicar la imagen de un indígena anclado a un tiempo mítico (Bonfill 1990, Albertani 1999). La relación entre la ciudad y el mundo indígena en los países andinos es antigua y no ha sido ajena ni a los indígenas de las zonas altas (Amodio 1996), tampoco a los indígenas amazónicos (Alexiades y Peluso 2015), ni al pasado ni la actualidad.

En las áreas andinas nos encontramos con numerosas ciudades que fueron constituidas por indígenas, independientemente de que la colonización marcase procesos de ruptura y de invisibilización de una población mayoritaria que fue minorizada (1), aunque los resultados de ese proceso han sido muy desiguales en las ciudades que han mantenido una base indígena mayoritaria, y en aquellas en las que como en Cuenca la colonización se tradujo en la recreación, primero, de un imaginario "blanco" y 
posteriormente mestizo, acompañada por la ruralización indígena. Lo cual no significa que lo indígena desapareciese de la ciudad, ni que esta pueda ser entendida al margen del contexto rural en el que se inserta. De hecho, las migraciones (ya sean temporales o permanentes) a la ciudad han sido una constante y se insertan habitualmente en estrategias colectivas y familiares -mantener actividades agroganaderas, reproducir determinados rituales, propiciar el que una parte del núcleo familiar acceda a estudios medios y superiores- posibilitando la articulación de la economía de subsistencia en la de mercado.

La vinculación entre los contextos rurales y urbanos es manifiesta en las migraciones interiores, mucho menos estudiadas en Ecuador que las migraciones internacionales que han contribuido a conformar lo que se ha denominado como comunidades transnacionales (2). Aunque los procesos migratorios implican transformaciones culturales y sociales, eso no significa necesariamente la desvinculación del migrante del contexto de procedencia. En todo caso, y pese al carácter tradicionalmente urbano de una parte de la población indígena y a que los procesos de migración a las ciudades se ha traducido habitualmente en una indigenización o reindigenización de las mismas, no deja de ser sorprendente su actual invisibilización en el caso cuencano.

Si hablar de modo genérico de "lo indígena" y de "la ciudad" puede contribuir a darnos una visión de conjunto sobre la situación de algunas minorías étnicas en relación a otras categorías racializadas (blancos, negros, mestizos...), también puede desvirtuar nuestra mirada si no tenemos en cuenta la diversidad de una denominación que se conforma como categoría de dominación para homogeneizar lo diverso, a partir de posiciones estructurales de desigualdad (Bonfill 1977). Por ello, es necesario profundizar en las diferentes estrategias culturales que son activadas por estos grupos, en función de la pertenencia étnica, en los contextos urbanos (Espinosa 2009). Y es que la emigración, entendida como hecho social (Figoli y Fazito 2009), tiene consecuencias diversas que pueden traducirse en la ocultación de los elementos que identifican a los grupos étnicos migrantes 0 , al contrario, en un proceso de reafirmación identitaria (Tamagno 2003, Varas 2005), en función de la constatación recursiva de las diferencias y de los niveles de aceptación o rechazo de la sociedad receptora.

Lo cierto es que si la situación estructural de desigualdad de los pueblos indígenas hace que encontremos elementos recurrentes en su inserción en la ciudad, también lo es que los grupos indígenas no se enfrentan de la misma forma a la realidad urbana, e incluso dentro de cada grupo étnico hay estrategias plurales en relación a la ocupación del espacio y a la resignificación de unos cuerpos, que permiten transitar desde la reafirmación de lo indígena a su negación. En este sentido, nos interesa analizar las estrategias utilizadas por tres grupos indígenas que viven en la ciudad Cuenca: cañaris, otavaleños y saraguros, centrándonos en sus niveles y formas de visibilización social. Partimos de la hipótesis de que etnia, género, clase social y edad son factores fundamentales a la hora de aproximarnos a una diversidad de estrategias habitualmente homogeneizadas en algunas investigaciones que conceptúan "lo indígena" como una realidad homogénea, partiendo de una falaz visión "comunitaria" que olvida que la comunidad es fundamentalmente un "deber ser", que habitualmente oculta las diferencias internas (Valcuende 1998).

Los tres pueblos seleccionados tienen toda una serie de denominadores comunes: todos ellos son kichwa-parlantes, han experimentado fuertes procesos migratorios en sus territorios de origen y tiene claros marcadores identitarios a partir de los que recrean su pertenencia étnica. Sin embargo, presentan algunas diferencias, como veremos posteriormente, en función de sus diversas especializaciones sociolaborales $\underline{(3)}$, entre otros factores.

El análisis de estas poblaciones indígenas nos permitirá comprender, partiendo de su caracterización socioeconómica: 1) las transformaciones experimentadas en las categorías de autoadscripción en el contexto urbano; 2) la creación y recreación de contextos de interacción en la ciudad; y 3) la reproducción de rituales ordinarios y extraordinarios a partir de los que se reafirman sus referentes de identificación. En definitiva, los procesos de ocultamiento o visibilización de sus referentes identitarios, partiendo de una noción de identidad de carácter relacional (Barth 1976).

\section{Metodología}


Este artículo es el resultado parcial de un trabajo de investigación en el que se han realizado 50 entrevistas a indígenas urbanos (4) y 15 entrevistas a agentes sociales vinculados con estos grupos a partir de la acción del propio Estado (5). Por otro lado, se ha trabajado con las estadísticas proporcionadas por el INEC (censos de población y vivienda del 2001 y 2010), que nos han permitido analizar la evolución en las categorías de autoadscripción y tener una visión global sobre las características socioeconómicas de estos grupos, siendo conscientes del valor relativo de una información en la que no se reflejan algunas migraciones estacionarias ni tampoco determinados tipos de trabajos. Una última técnica empleada está relacionada con la observación participante, que nos permitió aproximarnos a diferentes contextos de interacción de estos grupos: ámbitos festivo-ceremoniales, espacios de reunión y espacios de trabajo.

\section{Caracterización socioeconómica de las poblaciones indígenas en el cantón de Cuenca.}

Las cifras del Censo de Población y Vivienda-CPV (2001-2010) muestran una mayor proporción de población indígena en los cantones rurales de la ciudad de Cuenca. Sin embargo, en diez años el ritmo de crecimiento en esta área ha sido negativo $(-3,81 \%)$, produciéndose un leve crecimiento en las áreas urbanas $(0,49 \%)$. En todo caso, hay oscilaciones notables entre los diferentes grupos analizados. Los otavaleños tienden a crecer en las áreas urbanas del cantón. Una expansión vinculada a su actividad comercial, pero también a lo que parece una tendencia a la diversificación de actividades. Los cañaris crecen proporcionalmente de forma leve en el área urbana, mientras que la tendencia a la concentración urbana es especialmente marcada en los saraguros.

\section{Tabla 1}

\begin{tabular}{|c|c|c|c|c|c|c|}
\hline \multicolumn{7}{|c|}{ Cantón Cuenca. Otavaleños, cañarís y saraguros por área geográfica (\%) } \\
\hline \multirow{2}{*}{$\begin{array}{l}\text { Pueblo } \\
\text { Indígena }\end{array}$} & \multicolumn{3}{|c|}{ CPV 2001} & \multicolumn{3}{|c|}{ CPV 2010} \\
\hline & A. Urbana & A. Rural & Total & A. Urbana & A. Rural & Total \\
\hline Otavalo & 100.00 & 0.00 & 100 & 92.36 & 7.64 & 100 \\
\hline Cañarí & 17.88 & 82.12 & 100 & 19.97 & 80.03 & 100 \\
\hline Saraguro & 79.46 & 20.54 & 100 & 59.95 & 40.05 & 100 \\
\hline \multicolumn{7}{|c|}{ Fuente: Censo de Población y Vivienda 2001, 2010} \\
\hline
\end{tabular}

En el ámbito educativo, indicadores como tasa de analfabetismo, promedio de años de escolaridad (6) y niveles de instrucción, reflejan una brecha notable de la población indígena en relación a la media global. En 2010 la tasa de analfabetismo para la población indígena era del 15,14\% mientras que para los demás grupos sociales se sitúa en el 4,33\%. En diez años se ha producido un ligero descenso de esta tasa en la población indígena; no obstante, la reducción es menor a la de otros grupos sociales. Por otra parte, el promedio de años de escolaridad refleja 9,48 años lectivos aprobados para todos los segmentos poblacionales en 2010, mientras que el promedio para la población indígena se ubicó en 7,02 años.

Tabla 2 (7) 
Cantón Cuenca. Tasa de analfabetismo y promedio de años de escolaridad

\begin{tabular}{lcccc}
\hline \multirow{2}{*}{$\begin{array}{l}\text { Autoadscripcion } \\
\text { étnica }\end{array}$} & \multicolumn{2}{c}{$\begin{array}{c}\text { Tasa de } \\
\text { Analfabetismo (\%) }\end{array}$} & \multicolumn{2}{c}{$\begin{array}{r}\text { Promedio de años de } \\
\text { escolaridad }\end{array}$} \\
& CPV & CPV & CPV 2001 & CPV \\
& 2001 & 2010 & & \\
\hline Indigena & 16.18 & 15.14 & 4.33 & 7.02 \\
Negro & 7.63 & 3.92 & 6.74 & 8.31 \\
(Afrodescendiente) & & & 6.89 & 8.60 \\
Mulato & 6.13 & 3.78 & - & 8.55 \\
Montubio & - & 3.90 & 7.34 & 9.46 \\
Mestizo & 6.11 & 4.28 & 9.15 & 11.01 \\
Blanco & 2.92 & 2.02 & 8.06 & 10.31 \\
Otro & 4.55 & 3.61 & 7.43 & 9.48 \\
Total & 6.06 & 4.33 & &
\end{tabular}

Fuente: Censo de Población y Vivienda 2001, 2010

Entre los otavaleños, cañarís y saraguros se aprecian diferencias significativas. Los primeros muestran una tasa de analfabetismo superior a la media de la población indígena del cantón. Mientras que en los saraguros y cañaris se observa un descenso de la tasa de analfabetismo en el periodo intercensal 2001-2010, aunque crece este porcentaje en el caso de los otavaleños (8). En los tres casos analizados se aprecia un incremento en el promedio de años de escolaridad, siendo el pueblo cañarí el que registra un mayor progreso.

\section{Tabla 3}

Promedio de años de escolaridad de otavaleños, cañaris y saraguros en el Cantón Cuenca

\begin{tabular}{lcccccc}
\multirow{2}{*}{$\begin{array}{l}\text { Pueblo } \\
\text { Indigena }\end{array}$} & \multicolumn{2}{c}{$\begin{array}{c}\text { Tasa de A nalfabetismo } \\
(\%)\end{array}$} & & \multicolumn{2}{c}{$\begin{array}{c}\text { Promedio de años de } \\
\text { Escolaridad }\end{array}$} \\
\cline { 2 - 3 } \cline { 5 - 6 } & CPV 2001 & CPV 2010 & & CPV 2001 & CPV 2010 \\
Otavalo & 9.52 & 12.00 & & 5.11 & 6.63 \\
Cañari & 16.86 & 15.83 & & 3.67 & 6.93 \\
Saraguro & 8.39 & 5.25 & & 6.28 & 7.71
\end{tabular}

Fuente: Censo de Población y Vivienda 2001, 2010

Por niveles educativos, los saraguros muestran una mayor presencia en los niveles superiores. En cambio, los otavaleños y cañaris mantienen un porcentaje importante de población sin ninguna escolaridad, no apreciándose cambios significativos en diez años en el caso de los otavaleños. En posgrado, únicamente saraguros y cañarís están representados en el 2010.

\section{Tabla 4}




\begin{tabular}{|c|c|c|c|c|c|c|}
\hline \multirow{2}{*}{ Nivel de instrucción } & \multicolumn{3}{|c|}{ CPV 2001} & \multicolumn{3}{|c|}{ CPV 2010} \\
\hline & Otavalo & Cañarí & Saraguro & Otavalo & Cañań & Saragurc \\
\hline Ninguno & 10.14 & 16.10 & 4.76 & 10.42 & 12.48 & 3.98 \\
\hline $\begin{array}{l}\text { Centro de } \\
\text { Alfabetización/(EBA) }\end{array}$ & 0.00 & 0.91 & 1.19 & 0.69 & 2.16 & 0.57 \\
\hline Preescolar & - & - & - & 1.39 & 0.50 & 2.84 \\
\hline Primaria & 52.17 & 57.31 & 51.19 & 52.08 & 42.60 & 44.03 \\
\hline Secundaria & 8.70 & 7.19 & 20.83 & 12.50 & 11.73 & 13.07 \\
\hline Educación Básica & 18.84 & 2.97 & 7.14 & 10.42 & 18.05 & 15.91 \\
\hline $\begin{array}{l}\text { Bachillerato-Edu. } \\
\text { Media }\end{array}$ & 0.00 & 0.00 & 0.60 & 10.42 & 4.49 & 7.10 \\
\hline Ciclo Posbachillerato & 0.00 & 0.00 & 1.19 & 0.00 & 0.17 & 1.14 \\
\hline Superior & 2.90 & 2.51 & 7.14 & 2.08 & 6.24 & 9.38 \\
\hline Posgrado & 0.00 & 0.00 & 0.00 & 0.00 & 0.42 & 0.28 \\
\hline Se ignora & 7.25 & 13.01 & 5.95 & 0.00 & 1.16 & 1.70 \\
\hline Total & 100 & 100 & 100 & 100 & 100 & 100 \\
\hline
\end{tabular}

Fuente: Censo de Poblacion y Vivienda 2001, 2010

Como podemos apreciar, hay marcadas desigualdades de la población indígena en el ámbito educativo; en esto Cuenca no difiere de la dinámica global (pese a su porcentaje menor de analfabetismo). Sin embargo, las diferencias son significativas cuando nos aproximamos a los grupos seleccionados. Así llama la atención la menor presencia de otavaleños en niveles superiores de enseñanza en comparación a otros grupos. Un hecho posiblemente relacionado con la estrategia comercial, sustentada en una mano de obra familiar, que garantiza una pronta incorporación de los jóvenes al mercado laboral. Aunque no podemos olvidar que la emigración de saraguros y cañaris a Cuenca está motivada, en parte, por estudios (dada la proximidad del cantón del Cañar y en menor medida del cantón de Loja), a diferencia de los otavaleños que vienen con fines fundamentalmente de trabajo, lo que podría ser un dato distorsionador.

En el ámbito económico la tasa de ocupación global (TOG) (9), tasa de participación bruta (TPB) (10), tasa de desempleo (TD), categorías de ocupación, grupos de ocupación y ramas de actividad, evidencian algunas variaciones entre los diferentes segmentos poblacionales en el transcurso de los dos últimos censos. La TOG pasa del $98,76 \%$ en 2001 al $96,67 \%$ en 2010 . Por otra parte, la TPB pasa del $55,45 \%$ en el 2001 al $60.91 \%$ en el 2010 , una cifra solo superada por afrodescendientes y mulatos. La TD pasa de 1,24\% en 2001 a 3,33\% en 2010; cifra relativamente inferior al promedio general de 3,39\% para 2010.

Tabla 5

\begin{tabular}{|c|c|c|c|c|c|c|}
\hline \multirow{2}{*}{$\begin{array}{l}\text { Autoadscripcion } \\
\text { étnica }\end{array}$} & \multicolumn{2}{|c|}{$\begin{array}{l}\text { Tasa de Ocupación } \\
\text { Global-TOG }\end{array}$} & \multicolumn{2}{|c|}{$\begin{array}{c}\text { Tasa de Participación } \\
\text { Bruta-TPB }\end{array}$} & \multicolumn{2}{|c|}{$\begin{array}{l}\text { Tasa de Desempleo- } \\
\text { TD }\end{array}$} \\
\hline & $\begin{array}{l}\text { CPV } \\
2001\end{array}$ & $\begin{array}{l}\text { CPV } \\
2010\end{array}$ & $\begin{array}{l}\text { CPV } \\
2001\end{array}$ & $\begin{array}{l}\text { CPV } \\
2010\end{array}$ & $\begin{array}{l}\text { CPV } \\
2001\end{array}$ & $\begin{array}{l}\text { CPV } \\
2010 \\
\end{array}$ \\
\hline Indigena & 98.76 & 96.67 & 55.45 & 60.91 & 1.24 & 3.33 \\
\hline $\begin{array}{l}\text { Negro- } \\
\text { Afrodescendiente }\end{array}$ & 97.53 & 94.71 & 58.35 & 61.41 & 2.47 & 5.29 \\
\hline Mulato/a & 97.94 & 95.24 & 54.91 & 63.66 & 2.06 & 4.76 \\
\hline Monnuhio/a & - & 95.50 & - & 65.02 & - & 4.50 \\
\hline Mestizo/a & 98.17 & 96.71 & 50.09 & 56.30 & 1.83 & 3.29 \\
\hline Blanco/a & 97.64 & 95.86 & 48.41 & 54.67 & 2.36 & 4.14 \\
\hline Otro/a & 96.37 & 94.05 & 55.09 & 56.76 & 3.63 & 5.95 \\
\hline Total & 98.13 & 96.61 & 50.14 & 56.46 & 1.87 & 3.39 \\
\hline
\end{tabular}

Fuente: Censo de Población y Vivienda 2001, 2010

En los pueblos analizados, las TOG muestran en los dos periodos cierta similitud con respecto al 
promedio general para la población indígena de Cuenca. Con respecto a la TPB se observan marcadas diferencias entre estos pueblos en los dos periodos, a excepción del pueblo cañarí, cuya TPB es cercana al promedio indígena general para el cantón en los dos periodos. Las TPB del pueblo otavalo y saraguro son superiores al $70 \%$ en 2010 , lo que significa una participación mayor en el mercado laboral. Finalmente, la TD refleja un incremento para el 2010 con respecto al 2001, siendo el pueblo saraguro el que más aumenta con el $4,55 \%$, cifra relativamente superior al promedio indígena general para Cuenca.

\section{Tabla 6}

\begin{tabular}{|c|c|c|c|c|c|c|}
\hline \multirow{2}{*}{$\begin{array}{l}\text { Auloadscripcion } \\
\text { etnica }\end{array}$} & \multicolumn{2}{|c|}{$\begin{array}{l}\text { Tasa de Ocupación } \\
\text { Global-TOG }\end{array}$} & \multicolumn{2}{|c|}{$\begin{array}{l}\text { Tasa de Participación } \\
\text { Bruta-TPB }\end{array}$} & \multicolumn{2}{|c|}{$\begin{array}{l}\text { Tasa de Desempleo- } \\
\text { TD }\end{array}$} \\
\hline & $\begin{array}{l}\text { CPV } \\
2001\end{array}$ & CPV 2010 & CPV 2001 & $\begin{array}{l}\text { CPV } \\
2010\end{array}$ & $\begin{array}{l}\text { CPV } \\
2001\end{array}$ & $\begin{array}{l}\text { CPV } \\
2010\end{array}$ \\
\hline Otavalo & 100.00 & 97.94 & 85.71 & 77.60 & 0.00 & 2.06 \\
\hline Cañari & 99.54 & 98.13 & 57.25 & 55,12 & 0.16 & 1.87 \\
\hline Saraguro & 100.00 & 95.45 & 67.74 & 72.13 & 0.00 & 4.55 \\
\hline
\end{tabular}

Fuente: Censo de Población y Vivienda 2001, 2010

El análisis de estas cifras nos hace inferir que los más bajos niveles de escolaridad de la población indígena se explican en parte por su temprana incorporación al mercado laboral, lo que a su vez se refleja en las categorías de ocupación, grupos de ocupación y ramas de actividad predominantes, que en líneas generales podemos caracterizar como de escasa cualificación y baja remuneración.

Tabla 7

\begin{tabular}{|c|c|c|c|c|c|c|c|c|}
\hline $\begin{array}{c}\text { Categoria de } \\
\text { Ocupadión }\end{array}$ & Indigena & $\begin{array}{l}\text { Negro- } \\
\text { Afro- } \\
\text { ecuatoriano }\end{array}$ & Mulato & Montubio & Mestizo & Blanco & Otro & Total \\
\hline $\begin{array}{l}\text { Empleado u } \\
\text { obrero } \\
\text { Estatal }\end{array}$ & 5.81 & 8.61 & 8.90 & 11.08 & 12.12 & 12.14 & 8.64 & 11.91 \\
\hline $\begin{array}{l}\text { Empleado u } \\
\text { obrero } \\
\text { privado }\end{array}$ & 26.25 & 45.36 & 46.44 & 48.45 & 41.41 & 43.84 & 36.07 & 41.38 \\
\hline $\begin{array}{l}\text { Jomalero o } \\
\text { peón }\end{array}$ & 16.76 & 6.71 & 5.70 & 8.42 & 6.15 & 2.60 & 6.91 & 6.18 \\
\hline Patrono & 2.03 & 3.10 & 3.70 & 2.49 & 4.37 & 7.70 & 7.78 & 4.48 \\
\hline Socio & 0.57 & 1.51 & 1.99 & 1.03 & 1.61 & 2.50 & 1.94 & 1.64 \\
\hline $\begin{array}{l}\text { Cuenta } \\
\text { propia } \\
\text { Trabajador }\end{array}$ & 35.60 & 25.29 & 25.64 & 22.08 & 26.74 & 25.01 & 31.10 & 26.77 \\
\hline $\begin{array}{l}\text { no } \\
\text { remunerado }\end{array}$ & 2.63 & 1.16 & 1.71 & 0.60 & 1.44 & 1.25 & 1.51 & 1.45 \\
\hline $\begin{array}{l}\text { Empleado } \\
\text { doméstico }\end{array}$ & 7.34 & 5.30 & 3.42 & 3.09 & 3.50 & 2.20 & 2.59 & 3,53 \\
\hline Se ignora & 3.02 & 2.95 & 2.49 & 2.75 & 2.66 & 2.76 & 3.46 & 2.68 \\
\hline Total & 100 & 100 & 100 & 100 & 100 & 100 & 100 & 100 \\
\hline
\end{tabular}

Fuente: Censo de Población y Vivienda 2010

En cañaris, saraguros y otavaleños predomina la modalidad de trabajo por cuenta propia, empleado privado y jornalero o peón, con algunas diferencias considerables entre ellos.

Tabla 8 
Cantón Cuenca. Categoría de ocupación de otavaleños, cañaris y saraguros $=10$ años $(\%)$

\begin{tabular}{lccc}
\hline Categoría de Ocupación & Otavalo & Cañarí & Saraguro \\
\hline Empleado u obrero Estatal & 4.12 & 4.93 & 7.73 \\
Empleado u obrero privado & 17.53 & 29.76 & 28.64 \\
Jomalero o peón & 4.12 & 15.31 & 19.09 \\
Patrono & 3.09 & 2.55 & 2.27 \\
Socio & 2.06 & 1.19 & - \\
Cuenta propia & 51.55 & 29.08 & 28.18 \\
Trabajador no remunerado & 6.19 & 4.25 & 5.45 \\
Empleado doméstico & 2.06 & 8.84 & 4.55 \\
No declarado & 8.25 & 2.38 & 1.36 \\
Trabajador nuevo & 1.03 & 1.70 & 2.73 \\
Total & 100 & 100 & 100
\end{tabular}

Fuente: Censo de Población y Vivienda 2010

En los otavaleños hay una menor dispersión por ramas de actividad $(76,29 \%$ en el comercio -al por mayor y menor- y en industrias manufactureras). La población cañarí es la que refleja una mayor dispersión de su población, concentrando las actividades principales en labores agrícolas $(25,85 \%)$ y construcción (23,64\%). Finalmente, el comercio (al por mayor y menor), las labores agrícolas y la construcción constituyen las ramas económicas predominantes para los saraguro $(21,82 \%, 20,91 \%$ y $20,00 \%$ respectivamente). Un dato interesante es el relativo a la significativa presencia de los indígenas en el servicio doméstico en comparación a otros grupos. Esta ha sido una de las fuentes habituales de entrada a la ciudad y de trabajo para las mujeres indígenas (especialmente en el caso de las poblaciones cañaris), en un sector que se ha caracterizado por la informalidad, por lo que las cifras de los censos posiblemente son muy inferiores a las cifras reales $\underline{(11)}$.

\section{Tabla 9}

\begin{tabular}{lccc} 
Cantón Cuenca. Rama de actividad económica del pueblo indigena otavalo, cañari y \\
saraguro $\geq 10$ años (\%) & otavalo & cañari & saraguro \\
\hline Rama de actividad (Primer nivel) & 4.12 & 25.85 & 20.91 \\
\hline Agricultura, ganaderia, silvicultura y pesca & - & - & 0.45 \\
Explotación de minas y canteras & 36.08 & 11.39 & 7.27 \\
Industrias manufactureras & & & \\
Suministro de electricidad, gas, vapor y aire & - & 0.34 & - \\
acondicionado & - & 0.17 & 0.45 \\
Distribución de agua, alcantarillado y gestión de & 10.31 & 23.64 & 20.00 \\
deshechos & 40.21 & 11.90 & 21.82 \\
Construcción & 1.03 & 2.04 & 5.45 \\
Comercio al por mayor y menor & - & 2.04 & 2.73 \\
Transporte y almacenamiento & - & - & 1.36 \\
Actividades de alojamiento y servicio de comidas & - & 0.17 & 0.45 \\
Información y comunicación & - & 0.34 & 0.45 \\
Actividades financieras y de seguros & - & 1.87 & 2.27 \\
Actividades profesionales, cientificas y técnicas & 3.09 & 1.19 & 2.73 \\
Actividades de servicios administrativos y de apoyo & - & 3.23 & 1.82 \\
Administración pública y defensa & - & 1.36 & 1.82 \\
Enseíanza & - & 0.34 & 0.91 \\
Actividades de la atención de la salud humana & 1.03 & 1.02 & 0.45 \\
Artes, entretenimiento y recreación & 2.06 & 8.50 & 4.55 \\
Otras actividades de servicios & 1.03 & 2.89 & 1.36 \\
Actividades de los hogares como empleadores & 1.03 & 1.70 & 2.73 \\
No declarado & 100 & 100 & 100 \\
Trabajador nuevo & & & \\
Total & & &
\end{tabular}

Fuente: Censo de Población y Vivienda 2010

Finalmente, el análisis de los niveles de acceso a la seguridad social para los diferentes grupos sociales del cantón permite constatar la existencia de brechas considerables entre grupos étnicos, siendo la población indígena la que cuenta con menor cobertura, con el $15,80 \%$ que realiza aportaciones y el $0,48 \%$ de jubilados cubiertos por el sistema de seguridad social. 


\begin{tabular}{lccccc}
\multicolumn{6}{l}{ Cantón Cuenca. A filiación a la seguridad social = 18 afios (\%) } \\
\hline Pueblo Indigena & Aporta & $\begin{array}{c}\text { Es } \\
\text { jubilado }\end{array}$ & No aporta & Se ignora & Total \\
\hline Otavalo & 11.65 & 0.97 & 78.64 & 8.74 & 100 \\
Cañari & 16.52 & 0.37 & 81.63 & 1.48 & 100 \\
Saraguro & 15.15 & - & 83.12 & 1.73 & 100 \\
\hline
\end{tabular}

Fuente: Censo de Población y Vivienda 2010

Los otavaleños son los que tiene un menor nivel de cobertura en relación al promedio de la población indígena, mientras que cañaris $(16,52 \%)$ y saraguros $(15,15 \%)$ tienen niveles de cobertura cercanos al promedio general de la población indígena.

Tabla 11

\begin{tabular}{lccccc}
\multicolumn{6}{l}{ Cantón Cuenca. A filiación a la seguridad social = 18 afios (\%) } \\
\hline Pueblo Indigena & Aporta & jubilado & No aporta & Se ignora & Total \\
\hline Otavalo & 11.65 & 0.97 & 78.64 & 8.74 & 100 \\
Cañarí & 16.52 & 0.37 & 81.63 & 1.48 & 100 \\
Saraguro & 15.15 & - & 83.12 & 1.73 & 100 \\
\hline
\end{tabular}

Fuente: Censo de Población y Vivienda 2010

Los datos cuantitativos manifiestan, en líneas generales, una peor situación socioeconómica de los grupos indígenas urbanos en relación a otras categorías de autoadscripción, independientemente que las diferencias económicas internas entre ellos sean notables. Esto se evidencia no solo en la cualificación, el tipo de trabajo o el acceso a la seguridad social. El trabajo de campo nos ha mostrado también unas condiciones de vivienda en muchos casos claramente deficiente, en cuartos habitualmente compartidos y de reducidas dimensiones, situados bien en las áreas periféricas de la ciudad, en los denominados "conventillos" del centro urbano (12) o en áreas populares próximas a mercados como la Feria Libre. En el ámbito educativo, aunque se ha producido una significativa incorporación de los pueblos indígenas a la esfera educativa, la brecha en relación a otras poblaciones es evidente, especialmente en la gente de más edad.

\section{Cambios en los procesos de identificación}

El mestizaje en el caso ecuatoriano es un proyecto político y social del Estado para definir una sociedad de mezclas que busca, aparentemente, integrar diferentes mitologías 0 , si se prefiere, las diferentes historias en una historia oficial que, a su vez, supone una nueva forma de ocultación y jerarquización (Kingman 2002, 2003). Cuenca, que se ha definido por una estructura de clase marcadamente jerarquizada, también en base a criterios raciales, no ha sido ajena a estas transformaciones. La élite blanca fue progresivamente eclipsada por una élite mestiza, fruto en buena medida del proceso migratorio que emulaba los comportamientos de la antigua élite (Mancero 2013), lo que se ha evidenciado en un rejuego de identidades tal y como se manifiesta en los cambios en las categorías de autoadscripción socio-racial. 


\begin{tabular}{|c|c|c|c|c|c|}
\hline autoadscripción étnica & CPV 2001 & $\%$ & CPV 2010 & $\%$ & $\begin{array}{c}\text { Tasa de } \\
\text { crecimiento }\end{array}$ \\
\hline Indigena & 11163 & 2.67 & 9157 & 1.81 & -2.20 \\
\hline $\begin{array}{l}\text { Negro } \\
\text { (A frodescendiente) }\end{array}$ & 1171 & 0.28 & 8487 & 1.68 & 22.01 \\
\hline Mulato & 4092 & 0.98 & 2794 & 0.55 & -4.24 \\
\hline Montubio & - & - & 2144 & 0.42 & - \\
\hline Mestizo & 361008 & 86.44 & 453335 & 89.67 & 2.53 \\
\hline Blanco & 39520 & 9.46 & 28646 & 5.67 & -3.58 \\
\hline Otro & 678 & 0.16 & 1022 & 0.2 & 4,56 \\
\hline Total & 417632 & 100 & 505585 & 100 & 2,12 \\
\hline
\end{tabular}

Fuente: Censo de Población y Vivienda 2001, 2010

El primer hecho significativo es el importante descenso porcentual en diez años de la población que se define como "blanca". Esta reducción no puede explicarse en base a dinámicas demográficas, ya que Cuenca se ha convertido en una ciudad que está recibiendo un importante número de norteamericanos, mayoritariamente "blancos" (13). La reducción porcentual de las personas que se autoadscriben como "blancas" estaría relacionada con la progresiva tendencia de las clases sociales emergentes a definirse como "mestizas", a partir de una estrategia discursiva que se contrapone a la cerrada élite tradicional "blanca" (Valcuende y Vásquez 2016). Lo mestizo se ha convertido en el lugar políticamente correcto. El "mestizaje" es ahora también reivindicado por una parte de la clase media fenotípicamente blanca que tiende a desvincularse de la élite tradicional y para la que la blanquitud era, y en buena medida sigue siendo, un signo de distinción (14).

El descenso porcentual de la categoría blanco está acompañado por el aumento de la categoría "mestizo" y "afro", fenómenos que parecen interrelacionados. Una parte de los "mulatos" se han podido incorporar a dos categorías emergentes: mestizos y afros Los límites entre categorías son difusos y están fuertemente condicionados por factores sociopolíticos. En este contexto, la categoría "afro" es la que permite menos movilidad y la que presenta unos mayores niveles de estigmatización (15), aunque en los últimos años asistimos a una incipiente reivindicación del papel de los afros en la sociedad ecuatoriana, en general, y en la cuencana en particular.

El crecimiento de los mestizos es especialmente significativo, más si tenemos en cuenta que el reconocimiento de los "montubios" (no reflejado en el censo del 2001) debería traducirse en una reducción porcentual, como así ha sucedido en la zona de la costa donde su reconocimiento se ha traducido en la reducción de la primera categoría (16). En esta redefinición identitaria ¿qué ha sucedido con la población que se definía y se define como indígena? Nos encontramos con algunos datos aparentemente contradictorios. Si atendemos a los grupos estudiados, se produce un incremento porcentual de todos ellos (cañaris, otavaleños y saraguros); igualmente sucede con otros grupos indígenas concretos. La reducción porcentual, si analizamos los datos desagregados de los pueblos indígenas, afecta sustancialmente a "otros pueblos indígenas no determinados" (17). Parece razonable pensar que esta reducción se vincula con el crecimiento de categorías que se han ido visibilizando de forma progresiva (los grupos étnicos consolidados). En nueve años hemos asistido a un progresivo reconocimiento de nuevos grupos, pero también a una mayor centralidad del reconocimiento específico de algunos pueblos indígenas, lo que sin duda ha favorecido que personas que antes se reconocían como "indígenas" de forma genérica hoy pasen a identificarse a partir de una pertenencia étnica concreta, o bien pasen a formar parte de la reconocida categoría de mestizo. Sin embargo, aumenta el número de indígenas "ignorados" en el cantón Cuenca (18). Una posible interpretación de estos datos estaría vinculada al incremento del proceso de mestizaje entre los propios pueblos indígenas, fruto precisamente de la movilidad de población a la que hemos asistido en este periodo. De hecho, en las entrevistas realizadas entre matrimonios indígenas de diferentes pueblos encontramos serias dificultades a la hora de adscribir los hijos a una u otra etnia.

Estos datos indican un rejuego de categorías de autoadscripción que, como señalamos, solo puede ser explicado en función de una dinámica global que se traduce en la centralidad de la noción de mestizaje, pero también en la progresiva visibilización de grupos indígenas, que ocupan posiciones diversas en la 
ciudad a través de sus denominaciones específicas, en perjuicio de la categoría genérica "indígena". En todo caso, se observa un progresivo proceso de empoderamiento en relación al plural mundo mestizo, fragmentando entre los mestizos "blanqueados" y "los cholos".

- ¿De quién te enamorarías de una mestiza o de alguien de tu comunidad?

- La verdad no he pensado en eso. Creo que me da igual.

- ¿Y tu familia que te dice?

- Tampoco me dice nada, a veces me dicen no traerás una pollerona.

- ¿Te casarías con una pollerona?

- Con una pollerona, pollerona, no, con una mestiza sí, una saragura también, pero con una pollerona no, porque no me gustan las polleras. (Estudiante saraguro, 22 años).

En esta entrevista es interesante la diferenciación que se establece entre "mestiza" y "pollerona" (chola), que evidencia la segmentación de clase entre los mestizos, pero también un ascenso social de algunos grupos indígenas que hoy han accedido a unos ámbitos de poder superior al del reivindicado y al mismo tiempo denostado "cholo", subvirtiendo precisamente una categoría que en su tiempo supuso el reconocimiento de un estatus superior en relación a los indígenas (Mancero 2013). El proceso de empoderamiento identitario de los indígenas se evidencia especialmente entre la élite intelectual indígena que ha ido elaborando un discurso propio de puesta en valor de lo cañari, saraguro y otavaleño, en particular, y de lo indígena en general $\underline{(19)}$.

En todo caso, la identificación y autoidentificación en la ciudad conlleva reacciones diversas, vinculadas tanto con las características de cada uno de los grupos étnicos como por la situación del migrante en relación a su comunidad de origen. Así, a nivel general, en el discurso de una buena parte de nuestros informantes, la modernización con la que se vincula la ciudad se entiende como una pérdida de identidad, por lo que habitualmente se refieren a "la pureza" como un elemento distintivo identitario, tanto de los indígenas en relación a la sociedad mestiza (nosotros tenemos un pueblo, somos "puros") como entre los diferentes pueblos indígenas.

- ¿Crees que en la ciudad han recibido discriminación?

- No, para nada, tal vez hace años atrás, allí sí creo, ahora no, más bien nos valoran más, hay mayor reconocimiento, además por qué nos van a discriminar si nosotros los otavaleños somos algo puro, y eso me da mucho orgullo, en cambio, por ejemplo los de cuenca, son mestizos. (Otavaleño, 22 años).

El cambio del contexto rural al urbano implica necesariamente un proceso de readaptación, que al mismo tiempo supone generar un discurso legitimador que bien justifica la utilización de marcadores de identidad entendidos como tradicionales, o bien construir un discurso identitario que se reproduce a partir de nuevos marcadores, o incluso la negación de la vinculación identitaria con las comunidades de origen. En todo caso, nos encontramos con situaciones diversas que pueden implicar:

1) Una reafirmación identitaria reforzada por el contacto constante con las sociedades de origen. En estos casos, la emigración no supone una ruptura con el lugar de procedencia e ir a otro lugar se traduce en una mejora de las expectativas de vida, en muchos casos pensando en la posibilidad de regresar a sus comunidades de origen desde un estatus superior. El caso de los otavaleños es especialmente sintomático. Su estrategia económica, basada en la comercialización de productos con un marcado componente étnico, ha sido considerada como una estrategia comercial exitosa (Sobcyk y Soriano 2015) que, al mismo tiempo, ha facilitado su visibilización y la recreación de determinados componentes étnicos en las migraciones internacionales, pero también en las internas.

2) En algunos casos no se piensa en el regreso, y se percibe el trabajo o los estudios como un tránsito definitivo a la ciudad. La ciudad, por diversos motivos, se convierte en el destino, por lo que es necesario adaptarse a la nueva realidad. Este proceso implica un sentimiento de "pérdida de la identidad" que es vivido con sensaciones diversas, que van desde la resignación pasando por la melancolía o el alivio. En este último caso se hace hincapié en las mejores condiciones en el contexto urbano y en las dificultades de un regreso, tanto desde el punto de vista de las expectativas de vida, laborales, sentimentales, materiales... Esto es especialmente significativo en algunas mujeres entrevistadas, pero también en aquellos que por su forma de vida han preferido "exiliarse" de sus comunidades de origen. 
3) Un tercer grupo no se plantea su identidad. El carácter recursivo de la identidad está vinculado a un discurso letrado, a partir del cual lo étnico adquiere una nueva dimensión que, como veremos posteriormente, está muy vinculada tanto a las características generales de cada grupo como a las diferentes posiciones de clase de los propios indígenas.

Pero conviene detenernos en algunos factores que nos ayudan a explicar los procesos de reafirmación identitaria y las estrategias de invisibilización en función de factores como el género, la edad y la clase social.

\section{Estrategias de inserción de las poblaciones indígenas en el contexto urbano}

El desplazamiento desde zonas rurales a la ciudad obliga a redefinirse socialmente en un nuevo contexto de interacción, tanto en los referentes culturales usados en la vida cotidiana como en los marcadores identitarios que son activados u ocultados en función del contexto de interacción. Las entrevistas evidencian algunos problemas de adecuación, especialmente en la fase inicial de contacto con la ciudad, vinculados con los tipos de comida, el carácter más impersonal de las relaciones, la sensación de inseguridad, el tráfico, las dificultades de acceso a la vivienda. En este apartado nos centraremos en algunos elementos recurrentes en las entrevistas realizadas que nos permitan entender la activación de las prácticas y discursos identitarios de aquellos que, formando parte de la sociedad nacional, son considerados como "otros", en este caso internos.

\subsection{El género}

La primera diferencia significativa, recurrente en los pueblos analizados, es la distinción en función del género. Las mujeres experimentan un mayor peso de la tradición. Parafraseando a Marisol de la Cadena (1992), las mujeres son más indias. Si atendemos a dos criterios especialmente significativos de auto-representación, como el idioma o la forma de vestir, nos encontramos lo siguiente: las cifras del INEC (2010) para toda la población indígena del Ecuador manifiestan un mayor conocimiento de las lenguas originarias por parte de las mujeres. Este hecho se repite en el caso de los grupos étnicos analizados tanto en el ámbito rural como en el ámbito urbano del cantón Cuenca, aunque en este caso conviene hacer algunas precisiones. En 2010 hay 70 otavaleños que hablan quechua (49\%), reproduciéndose nuevamente la diferencia de género (56\% mujeres y $49 \%$ hombres). En el caso de los cañaris, 299 personas (25\%) hablan quechua, reduciéndose su número en relación al 2001 (57\% mujeres y $43 \%$ hombres). El porcentaje se reduce en los saraguros urbanos: 54 hablan su lengua (15\%), un grupo en el que la tendencia global en relación al mayor peso de las mujeres se invierte en favor de los hombres ( $41 \%$ y $59 \%$ respectivamente).

Igualmente sucede con la utilización de la ropa tradicional. La observación directa pone de manifiesto una tendencia mayor en las mujeres a usar sus vestidos tradicionales de forma cotidiana en comparación con los hombres.

"Para mí es tan importante (la ropa) porque es mi cultura, desde pequeñita me enseñaron mis padres a usar su misma vestimenta. Por eso tengo que respetar mi cultura y por eso yo digo que dejare con la muerte mi cultura...". (Cañari, estudiante, 32 años).

El dejar de utilizar las ropas tradicionales ha sido más penalizado socialmente en las mujeres que en los hombres.

- (A mis hijos) a veces les hago vestir como nosotros, como otavaleños, con pantalón blanco así,

- ¿Generalmente cuando se visten así?

-Cuando hay un compromiso

- ¿En qué compromiso generalmente se usa el traje?

- Para todo compromiso mismo utilizamos mismo el pantalón blanco. Hasta mi esposo no le gusta poner así, dice que, claro que el pantalón blanco es bien manchoso y toca cuidar como si fuera algo jajaja... y así mismo alpargatas también. Entonces así ellos ponen en algún compromiso, hay sí

- ¿Y su hija de 27 años, ella ocupa el traje?

- Ella sí iqué vamos a cambiar!, cambiar, cambiar, ¡nunca! Nosotros nuestra ropa típica para que 
vamos a cambiar. Claro que cuando son pequeños ponen cualquier ropita, pero ellos ya tienen manera de vestir como nosotros. (Otavaleña, comerciante, 47 años).

Las razones esgrimidas por las mujeres que han cambiado los anacos o las polleras por los jeans, están relacionadas con el precio de la ropa. Es más barato comprar un pantalón que el anaco. El argumento en parte es cierto y en parte no. Lo es porque la ropa de gala efectivamente puede llegar a suponer un desembolso económico importante (aunque como podemos observar en las cañaris los vestidos también se han ido adecuando y modificando con telas de menor precio), pero también a la comodidad, ya que es preciso que las mujeres habitúen su cintura desde niñas para llevar estas prendas. En otros casos se argumenta que prefieren pasar desapercibidas o incluso el gusto por las ropas occidentales.

- ¿Utilizas siempre el traje?

- No, el primer año sí pasaba con mi traje, pero ya después el hecho de ser indígena implica que tu ropa es cara: las blusas, las polleras, los collares (...). Cuando voy a mi comunidad siempre voy con mi vestimenta, yo siempre me siento orgullosa de ser indígena. (Estudiante de Universidad, 24 años, cañari).

Hay una progresiva transformación de las ropas tradicionales que incorpora telas más cómodas y más baratas, como es especialmente apreciable en las mujeres cañaris. Pero también hay una tendencia a considerar los trajes tradicionales como elementos excepcionales, utilizados solo en los acontecimientos especiales. El traje "indígena" progresivamente se va transformando en un traje de gala, por lo que se usa en los momentos de reafirmación identitaria.

En el caso de los hombres, su visibilización no pasa tanto por la ropa, que pone de manifiesto la pertenencia étnica concreta, como por el mantenimiento del "huango". De hecho es bastante habitual que los indígenas de los diferentes grupos tiendan a no utilizar sus ropas tradicionales, aunque mantienen el huango como elemento distintivo.

\subsection{Edad}

Al igual que sucede con el género, la edad constituye un elemento importante que evidencia las transformaciones experimentadas por los diferentes grupos étnicos. Así a nivel nacional, según los datos del INEC para toda la población indígena, se aprecia que la proporción de hablantes se incrementa en los grupos de mayor edad (en promedio a partir de los 20 años para los tres pueblos analizados) (20). La pérdida del idioma propio está relacionada con un periodo histórico de ocultamiento de las señas de identidad (21), asociadas con un mundo de atraso, pero tal y como hemos podido observar a través del trabajo de campo en la ciudad, igualmente sucede con la forma de vestir. En las mujeres y hombres más jóvenes es habitual que tiendan a no utilizar las ropas tradicionales. La inserción en las instituciones escolares supone un momento crucial en el que los jóvenes indígenas son conscientes de sus diferencias. El huango que era un símbolo de pertenencia étnica, pero también de masculinidad, es traducido en la sociedad mestiza como rasgo de feminidad, por lo que en el periodo de la adolescencia es bastante frecuente que los jóvenes se corten el huango, aunque en líneas generales hay un número importante de hombres que visibilizan su cuerpo como indígenas a partir de este elemento cultural.

"Mi guagua como tenia cabello, yo mandaba haciendo trencita, siempre acostumbramos en campo, los hombres tienen trenza, entonces, primero yo vine a dejar a la escuela, y quedo con el uniformecito. Con el uniforme sí pero con el pelo no, el pelo no hago cortar, entonces, que es lo que pasó, cuando yo llego tarde para retirar, primer día de clases, allí como que medio "agachaditu", triste, dice: mami yo no quiero estar aquí, yo digo ¿Por qué Carlitos? ¿Por qué mijo? ¿ (...), y él ya dice, mami quieres que quede aquí tienen que hacer cortar pelo sino mándame a la escuelita que yo estudiaba (él estudiaba (...) en la comunidad mismo). Yo quiero ir allá, dice, porque aquí a mí me hacen lado, yo no tengo amiguitos como tenía en Cañar, en mi escuelita no tengo amigos, porque hasta... hasta que me fui al baño se fueron siguiendo, porque decían que soy mujer, que llevaba mi cabellito, entonces... le... no tengo amigos... todos hacen a lado, todo se ríen de mí, que todos dicen que soy mujer, mis amigos decían que soy Juanita (22), él llama... Ilama Juan, allí si fue bastante duro... yo le lloré cuando... me contó esto... pues él dijo mami... yo no quiero estar aquí, yo quiero ir a Cañar, y de allí yo dije: no mijito al siguiente día, yo dije mijo no..., decirte primeramente por qué no puedo hacerte cortar pelo... porque es nuestra tradición, nuestra cultura por eso yo no... no puedo hacer cortar, claro que nadie no viene a decir usted no debe cortar pelo, es mi propia decisión, o 
sea... Yo me valoro mi cultura, mi tradición, entonces a mí enseñó mis padres así y yo también me valoro y enseño a mis hijos así". (Cañari, 32 años).

A su vez, en el caso de las mujeres la utilización de algunas prendas, como el anaco, supone un proceso de aprendizaje que se inicia en la infancia. La uniformización en las instituciones escolares, y un nuevo contexto en el que es más cómodo utilizar otro tipo de ropas, contribuye a que las más jóvenes dejen de utilizar un tipo de ropa que cada vez ha ido perdiendo valor en la vida cotidiana aunque, como ya señalamos, conserve su importancia en contextos festivo-ceremoniales.

Sin embargo, no solo es la forma de vestir la que marca diferencias entre generaciones. Es interesante observar cómo algunos de estos jóvenes se identifican con otra realidad diferente a la de sus padres en función de un nuevo contexto espaciotemporal (23), haciendo de la música un referente de identificación de una enorme importancia, a partir del que la juventud indígena reivindica su singularidad, precisamente en función de un "lenguaje" que les permite articularse con la sociedad global.

"Antes mi abuelita, sí me decía acerca de la forma de vestir, cuando yo me corte el cabello, hace dos años que estuve en Chile, me hice el corte 'emo'. Mis familiares me vieron por facebook, y me decían que parezco el diablo, que no se ve bien, está feo, le hacían chistes, pero no es que lo discriminaban (...) se reían. (...) Me gusta la moda, la cultura de los indios americanos cheroquis. Algunos compañeros utilizan aretes de plumas y otros de plata como piedras, por la cultura rapera. El reggaetón me encanta, también la bachata, pero otras no tanto, la música folclórica realmente la escucho, pero no me gusta tanto (...). Un ratito sí puedo escuchar, como la Chica Otavaleña, pero no escucho mucha música folclórica". (Otavaleño, 22 años).

\subsection{El sector de actividad y clase social}

Los referentes culturales singulares adquieren un carácter recursivo especialmente para las élites indígenas urbanas y la población que se ha formado en las universidades. No podemos olvidar que los liderazgos en estos nuevos contextos devienen en buena medida de la autoridad y/o el poder efectivo en sus propias comunidades, pero también de la capacidad de mantener esa singularidad en una sociedad pluricultural, haciendo de "puente" entre los contextos globales y locales. No es extraño que la visibilización de estos grupos sea clara, también como una estrategia de reivindicación identitaria, especialmente en los organismos vinculados con la administración, o en sectores relacionados con la educación o la cultura. Sin embargo, no podemos olvidar que este proceso es reciente y que hasta hace no mucho tiempo los testimonios de discriminación en la propia universidad eran claros y habituales, tal y como se pone de manifiesto en diversas entrevistas realizadas, en las que se expresa, por ejemplo, las burlas de compañeros y profesores, y los conflictos producidos por acudir a las clases con sus ropas tradicionales.

"María [nombre supuesto], joven indígena estudiante de derecho, por los años 90 asistía a clase con su vestimenta completa incluido el sombrero, algunos profesores le habían indicado que no puede estar en clase con el sombrero, pero ella no les hizo caso de manera que siguió con el sobrero. Estando ya en el último año de estudios un profesor le dijo que tenía la obligación de quitarse el sombrero, al menos en las clases que recibía con él porque seguir con el sombrero era mala educación, ella le dijo: 'el sombrero es parte de mi vestimenta, entonces pedir que me quite es lo mismo que yo le obligara a usted que se quite su saco porque darme clase con él es faltar al respeto, tanto a mí como al resto de mis compañeros'. María terminó la carrera de abogada pero se graduó después de casi cinco años de terminar. Por miedo a que ese profesor fuera miembro del tribunal, tuvo que esperar a que se jubilase". (Profesora de la Universidad de Cuenca).

En otros sectores de actividad la situación es diversa, siendo las actividades con una fuerte presencia indígena (actividades agrarias en las parroquias rurales del cantón o actividades comerciales en mercados populares) donde el nivel de visibilización es mayor. En este sentido, debemos destacar la centralidad de los mercados como ámbitos de trabajo y de sociabilidad para una buena parte de estos pueblos. Sea la Feria Libre, que concentra a un grupo importante de comerciantes, estibadores y vendedores ambulantes (cañari y saraguro, principalmente) o en el centro de la ciudad, donde se hacen especialmente presentes los otavaleños que venden sus productos en áreas como la plaza de San 
Francisco; una zona central desde el punto de vista geográfico, pero con ciertas connotaciones negativas para la clase media, en cuanto que, por un lado, continúa siendo el ámbito de encuentro de hombres que buscan trabajos temporales; por otro, es un contexto que conecta el mundo rural con el urbano, como se pone de manifiesto en el tipo de tiendas y establecimientos de carácter marcadamente popular, cuyos servicios son especialmente importantes para la población rural del cantón (24). En áreas próximas del corazón de la ciudad, como el parque Calderón, no era extraño hasta hace poco tiempo, incluso en la actualidad, ver la presencia de niños Saraguros que ofrecen sus servicios de lustrabotas, especialmente en los periodos festivos y vacacionales.

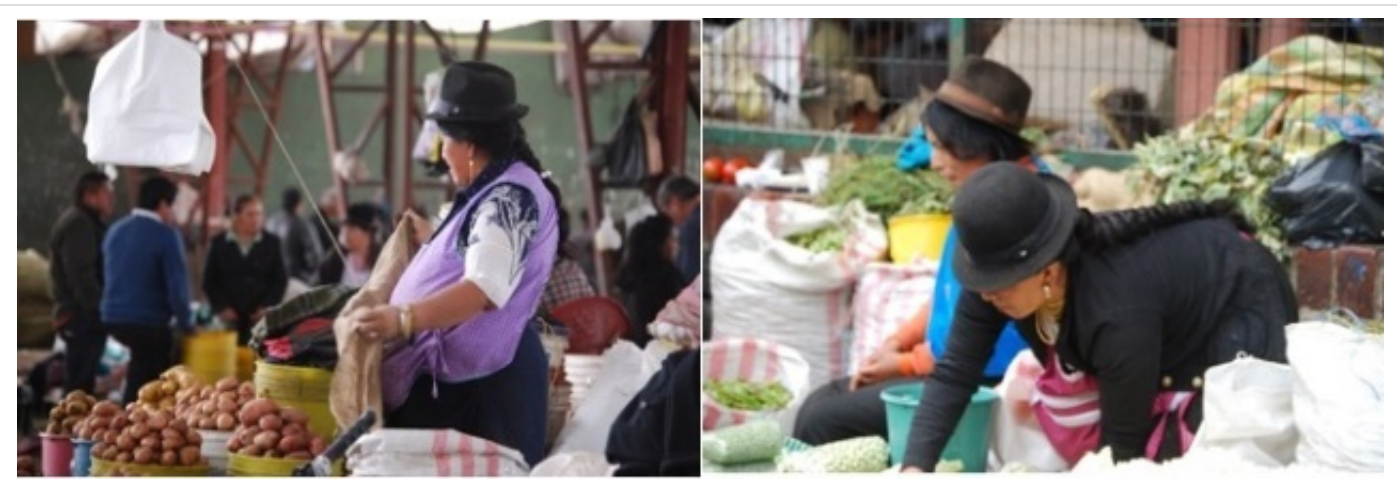

Mercado Feria Libre. Fotografía: José Luis Fernández Sánchez, 2014

La vinculación entre actividades y espacios concretos, donde se asiste a una cierta especialización laboral, son elementos que parecen favorecer la reproducción de los marcadores identitarios, particularmente en ámbitos como el comercio (en los que se asiste incluso a una cierta especialización por grupo étnico en función de los productos) en los que las mujeres tienen una presencia y centralidad especialmente significativa. Otra cuestión diferente es en los contextos en los que los indígenas deben entrar a formar parte de ámbitos que funcionan desde estándares blanco-mestizos, en los que sí es habitual que se tiendan a utilizar estrategias de invisibilización.

"Alberto que trabaja como administrativos señala que no sufre discriminación, sin embargo en otra pregunta reconoce que cuando se busca trabajo habitualmente se oculta la procedencia". (Administrativo cañari, entrevista no grabada, 2014).

Un mismo hecho social puede responder a diversas causas, así comprobamos que es entre los sectores populares y aquellos que ocupan posiciones relevantes de poder, dónde la visibilización de los indígenas es más clara. Las nuevas elites recrean unas señas de identidad que les permite reafirmar su posición en un mundo en cambio, como así sucede en el ámbito educativo y cultural. Para los sectores populares la identidad no es una opción, especialmente para los mayores. No es extraño que, en este último caso, los referentes identitarios indígenas se vinculen con una situación de pobreza y que los referentes de identificación adquieran un marcado carácter instrumental.

- ¿Usted sabe quechua?

- Sí

$-¿ Y$ sus hijos?

- No

- ¿Y no le gustaría que aprendiesen?"

- Prefiero que hablen inglés que sirve para comunicarse con más gente. (Saraguro, entrevista informal no grabada).

\section{Contextos de interacción y acciones simbólicas}

En nuestra búsqueda de la ciudad indígena, uno de los objetivos era identificar contextos festivoceremoniales de reproducción identitaria de los diversos grupos analizados. La realidad es que la presencia en estos contextos festivo-ceremoniales es en todos los grupos limitada, pero además pone en evidencia las marcadas diferencias sociales existentes en los grupos étnicos estudiados. 
La participación en contextos festivo-ceremoniales de saraguros y cañaris es escasa. Sus espacios de interacción étnica se mueven habitualmente en contextos privados, aunque en algunos casos su presencia es notoria, como así sucede en el caso de "los pases del niño", vinculados a la Feria Libre. Los espacios de sociabilidad señalados por los indígenas se vinculan a: zonas urbanas próximas a las áreas de trabajo, algunos parques periféricos y, en el caso de los varones, actividades relacionadas con el fútbol.

Durante el tiempo que duró la investigación el equipo estuvo presente en dos celebraciones, con un cierto carácter iniciático, de recibimiento de los nuevos estudiantes en la Universidad de Cuenca. En estos contextos se recrea la pertenencia étnica, especialmente a partir de la música, pero hay dos elementos que nos merecen una especial atención. El primero es lo que no se ve, y está relacionado con la ausencia de saraguros o cañaris pertenecientes a sectores sociales subalternos. El segundo es que, aunque hay un predominio del grupo étnico que organiza la celebración, también es habitual la presencia de otros indígenas universitarios (las relaciones son especialmente claras entre saraguros y cañaris); el contacto en la universidad favorece la interacción entre indígenas de diversas etnias.

Solamente en el caso de los oavaleños encontramos una significativa participación en algunos contextos asociativos y festivo-ceremoniales, con un claro componente étnico, articulándose espacio-grupoactividad. En la plaza de San Francisco donde este grupo es especialmente visible en función de la actividad comercial juegan un papel protagonista en momentos cotidianos y extraordinarios, como en "el pase del niño" y en "los viejos" (25). Detenernos en la organización de este acto durante la navidad del 2013, nos puede dar claves para entender la reproducción identitaria de este grupo y las diferencias con los otros grupos indígenas urbanos.

La escenificación representada para ser quemada no hacía referencia a ningún acontecimiento nacional o local; se había elegido un hecho acontecido en Otavalo: la polémica ordenanza municipal que prohibía a los peluqueros rurales cortar la trenza a menores de edad, sin autorización de los padres. No deja de ser sorprendente las referencias al lugar de origen, con el que mantienen constantes relaciones familiares y comerciales. Los otavaleños tienen la articulación suficiente e intereses comunes que sustentan una fuerte identidad, que reproducen en la ciudad de Cuenca. Sin embargo, cuando preguntamos a saraguros y cañaris sobre su escasa visibilidad, señalan que la cercanía y vinculación con sus lugares de origen lo hace innecesario.

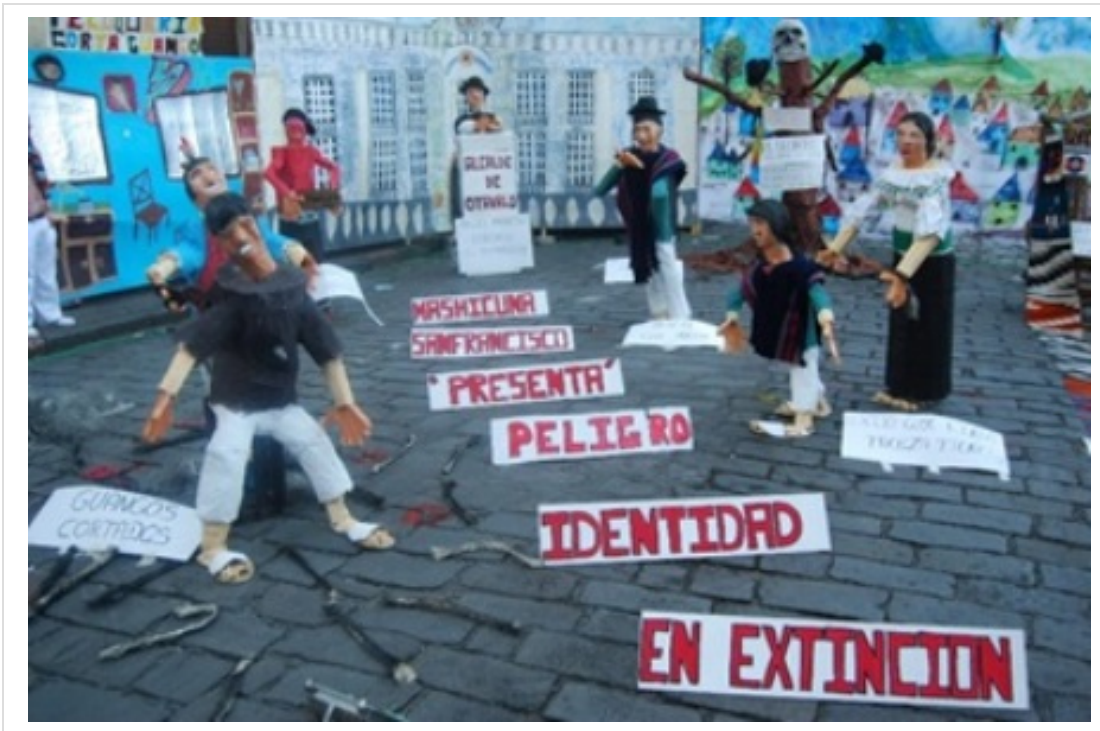

“Quema del Viejo". Plaza San Francisco, 2013. Fotografía: José Luís Fernández Sánchez

Como vemos, los contextos de sociabilidad y trabajo están marcados por la etnia pero también por posiciones de clase, que en la cotidianidad de la ciudad fragmentan las relaciones de solidaridad que se producían en el ámbito rural. Un último aspecto reseñable, en relación a los espacios en los que los indígenas encuentran ámbitos para la reproducción de su identidad, tiene que ver con el carácter marcadamente popular de los mismos, lo que nos indica también la segregación socioespacial de los contextos urbanos. 


\section{Conclusiones}

Ser indígena y urbano son realidades que han sido consideradas discursivamente como incompatibles hasta hace muy poco tiempo. En Ecuador, el indígena representa en el imaginario lo contrario a la ciudad. Las ciudades se definieron como blancas o mestizas pero no como indígenas. De hecho, llama la atención el doble alejamiento discursivo que se produce en relación al indígena: temporal y espacial. La imagen del indígena ha remitido siempre al pasado, en el caso de Cuenca a los yacimientos arqueológicos donde se rememora la presencia cañari. El indígena es para la sociedad dominante una imagen atemporal, por lo que cualquier cambio se define en función de "dejar de ser". El indígena no es por tanto presente y mucho menos urbano. Y aquí entramos en el segundo alejamiento discursivo (espacial). Ser indígena básicamente se definía por estar vinculado al campo, es decir por la ruralidad, ámbito donde se mantienen viejas y nuevas esencias, que en el caso del indígena podían ser leídas en clave de "salvajismo" o en clave de inocencia primigenia, o en ambas claves al mismo tiempo. Sin embargo, a partir de la década de los noventa asistimos a un resurgir de los movimientos afro e indígenas en América Latina, en general, y en Ecuador en particular. Toda una serie de transformaciones económicas, vinculadas a los importantes procesos migratorios experimentados por la sociedad ecuatoriana, unidos al resurgir político de los movimientos indígenas permitieron la conversión del indígena en sujeto político; el caso de Cuenca no es una excepción.

La investigación realizada evidencia algunos aspectos importantes que es preciso recapitular. El primero es que, pese a ser numéricamente mucho menos significativos, la presencia de los otavaleños es realmente importante en el contexto urbano en base a dos elementos. El primero, su identificación con una actividad comercial que les permite articularse tanto a nivel espacial, en ámbitos espacialmente centrales, aunque socialmente considerados periféricos de la ciudad, como a nivel asociativo, un elemento que ha contribuido a una notable presencia de este grupo en contextos festivo-ceremoniales. La imagen del otavaleño rompe en cierta medida el imaginario del indígena, por lo que no es casual que pese a esta visibilización a nivel discursivo su presencia no sea destacada en las entrevistas realizadas a los técnicos que trabajan con población indígena. $Y$ es que los oavaleños son reconocidos más como otavaleños que como indígenas, tanto por su región de procedencia como por el temprano proceso de reconocimiento de este grupo, y por una posición socioecónomica, política e incluso espacial más definida en relación a otros grupos, que de alguna u otra forma se vinculan a la ruralidad y a un estatus inferior, más "propio" de indígenas. La actividad económica desempeñada por unos y otros grupos parece jugar un papel central en sus niveles de visibilización. Este hecho se reproduce en el caso de los saraguros y de los cañaris vinculados a lo que son auténticos puentes que conectan el mundo urbano y rural: los mercados. Es precisamente en estos contextos en los que estos grupos se visibilizan como indígenas. No es casual que sea la actividad comercial la que juegue un papel importante en la reproducción de determinados elementos estéticos, y no lo es por tres razones: 1) el protagonismo de las mujeres y el papel jugado por la familia en este tipo de negocios; 2) la importancia de identificar determinados productos con las bondades de su origen o su forma de producción, rural y/o indígena; y 3) el carácter central de esos espacios en la vida social urbana, unido a unas ciertas connotaciones periféricas, en cuanto puente que une el mundo urbano y rural.

En otros contextos las estrategias de visibilización son más dispares. En este sentido no podemos olvidar el papel uniformizador de los contextos educativos, pero también el desigual valor de lo indígena en unos y otros contextos, en los que puede ser un elemento estigmatizador o bien un elemento que garantiza una presencia social significativa, como así ha empezado a suceder, por ejemplo, en la Universidad, donde se hacen especialmente presentes cañaris y saraguros.

En todo caso el mantenimiento de los referentes de identificación étnicos están vinculados con la edad y con la clase social. Los más jóvenes tienden a buscar en algunos casos otros referentes identitarios, como la música, o bien a invisibilizarse, especialmente aquellos que rompen sus vinculaciones con las comunidades de origen o que pertenecen a comunidades transnacionales que han adoptado los valores de la sociedad global, como se pone de manifiesto en algunas comunidades indígenas. 


\section{Notas}

1. En el caso ecuatoriano, los primeros registros poblacionales realizados durante el periodo colonial e inicios del periodo republicano reflejan la presencia de una sociedad mayoritariamente indígena, cercana al $70 \%$ de la población total. Actualmente esta población ocupa un porcentaje mínimo (7\%), si lo comparamos con países como Bolivia (62\%), Guatemala (41\%), y Perú (32\%). Ecuador sería uno de los países que ha experimentado un proceso de mestizaje más intenso de América Latina (Hidekazu 2011). La configuración y posterior consolidación del Estado mestizo a mediados del siglo XX, que reivindica el mestizaje como parte consustancial de la identidad nacional, nos ayudan a entender en parte este hecho social.

2. Para un análisis más pormenorizado de este concepto véase Canales y Zlolniski 2001.

3. Los cañaris son los que han mantenido una vinculación histórica más importante con Cuenca. Geográficamente se sitúan en el cantón más próximo a esta ciudad: el Cañar. Los saraguros se encuentran algo más alejados, en el cantón de Loja (aunque su presencia es importante en otros cantones) y por último los otavaleños provienen de la región más alejada, situada al norte del país: Otavalo.

4. Se han seleccionado tanto informantes que se autodefinen como "indígenas" y que son los mayoritarios, como personas que aunque no re reconocen como tales, son categorizados así por otros grupos de autoadscripción.

5. Este trabajo se ha realizado con el patrocinio de la Secretaría de Educación Superior, Ciencia, Tecnología e Innovación de la República del Ecuador (SENECYT), a la que agradecemos su patrocinio, junto al apoyo recibido por la DIUC de la Universidad de Cuenca. El equipo del Aula de Derechos Humanos que se encargó de la recopilación de la información, además de los firmantes de este artículo, estuvo compuesto por: Nilsson Araujo, Ángel Rodrigo Japón, Bolivar Ávila, Alberto Chaves, Nataly Cajamarca, Daysi Ayala y Mónica Novillo, a los que se puede considerar como coautores de este trabajo.

6. Promedio de años lectivos aprobados de la educación formal, en los niveles básicos, medio, posbachillerato, superior y posgrado a partir de los diez años.

7. Esta categoría étnica (montubio) de autoadscripcion no formaba parte de la boleta censal del 2001, por lo que no existen registros para este periodo. Los montubios son campesinos situados en la costa ecuatoriana. Fueron reconocidos como pueblo por la Constitución ecuatoriana en 2008. Para un análisis más detallado sobre este pueblo véase Rivadeneira 2012. La categoría montubio, como referente identitario surge a partir del propio proceso de mestizaje pero reinvindicando su singularidad con respecto a la identidad mestiza en general, definiéndose a sí mismos como: "El resultado de un complejo proceso histórico de adaptación y transformación étnica, interregional y socio-cultural que se dio en el litoral donde se fusionaron indios, blancos y negros; de esta fusión provenimos los montubios, con cultura, identidad propia y particular realidad, que nos diferencia de mestizos y campesinos de la sierra y otros pueblos del país" (Hidekazu 2011: 48).

8. Pese al descenso porcentual en los tres grupos, en el último censo se observa un crecimiento en el número total de analfabetos indígenas en el área urbana.

9. Peso porcentual de la población ocupada con respecto a la económicamente activa (PEA).

10. Población Económicamente Activa (PEA) con respecto a la población total.

11. Muchas mujeres cañaris de escasos recursos comenzaban trabajando de niñas en la casa de los patrones o de personas conocidas, en régimen de internado. En muchos casos no había remuneración económica, en una posición marcada por la subsidiaridad y las relaciones de poder.

12. Los "conventillos" son el equivalente español de "patio de vecinos" o "casa de vecinos". La denominación de "conventillos" se utiliza en otros países de América Latina además de Ecuador. En el caso cuencano se sitúan en el centro urbano y suelen ser grandes casas que fueron compartimentadas por una elite venida a menos, siendo alquilados los diferentes cuartos que pasan a ser ocupados por los sectores populares. 
13. A nivel nacional la población blanca refleja una tasa negativa de $-4,06 \%$ en el periodo intercensal 2001-2010, lo que se refleja en la reducción de su peso relativo, pues pasa del $10,46 \%$ en el censo del 2001 al 6,09\% en el de 2010.

14. Para Viveros (2010: 19), "el espejismo de la blanquitud no marcada" es un ámbito que pese a su centralidad apenas ha sido explorado.

15. Las entrevistas realizadas a técnicos de diversas administraciones señalan al grupo "afro" como especialmente estigmatizado en la sociedad local.

16. El peso relativo de la población mestiza en la región costa desciende de $78,44 \%$ en 2001 a $66,33 \%$ en 2010 , lo que a su vez se corrobora por la tasa de crecimiento del $0,12 \%$.

17. La variación demográfica es drástica en el periodo analizado. En efecto, la proporción de esta categoría con respecto a la población indígena pasa de 74,74\% en el Censo del 2001 a 0,64\% para el Censo del 2010 (tasa negativa de $-55,02 \%$ ).

18. La proporción de la población de pueblos y nacionalidades que se ignora pasa de $1,17 \%$ en el censo del 2001 al 40,49\% en el de 2010, lo que se representa una tasa de crecimiento del $37,15 \%$.

19. Para un análisis de los procesos de conformación de la "neo-indianidad" en América Latina véase Galinier y Molinié 2013.

20. En el Cantón Cuenca nos encontramos nuevamente con un mayor conocimiento de la lengua originaria en función de la edad, aunque el porcentaje de hablantes es mínimo en el rango de edad comprendido entre 5 y 14 años (en promedio: 9,43\% otavaleños, $12,51 \%$ cañaris y $5,94 \%$ saraguros).

21. Son numerosos los testimonios que señalan como los padres evitaron que los hijos aprendiesen el quechua.

22. Hemos modificado el nombre para preservar la intimidad del niño.

23. Vásquez (2014) analiza en una interesante investigación las transformaciones experimentada por la juventud cañari a partir del proceso de migración y la experiencia transnacional.

24. Los mercados han jugado un papel central como ámbito de interacción de las diferentes poblaciones indígenas y no sólo de los grupos analizados, tanto en Cuenca como en otras ciudades andinas. Para el caso de Cuenca véase Mancero 2013, y para el caso de Quito, Cuminao 2012.

25. Los viejos se celebran la última noche del año. Se realiza una escenografía con personajes y situaciones que representan acontecimientos importantes del año, que al final es quemada.

\section{Bibliografía}

Albertani, Claudio

1999 "Los pueblos indígenas y la ciudad de México. Una aproximación”, Política y cultura, n 12 : 195-221.

Alexiades, Miguel (y Daniela Peluso)

2015 "Introduction: Indigenous Urbanization in Lowland South America", The Journal of Latin American and Caribbean Anthropology, $\mathrm{n}^{\circ}$ 20: 1-12.

Amodio, Emanuele

1996 "Los indios metropolitanos: identidad étnica, estrategias políticas y globalización entre los pueblos indígenas de América Latina", en Montero Mato y E. Amodio (coords.), América Latina en tiempos de globalización. Caracas, UNESCO/Asociación Latinoamericana de Sociología/UCV: 51-66.

Barth, Fredrik 
1976 Los grupos étnicos y sus fronteras. México D. F., Fondo de Cultura Económica.

Bonfil Batalla, Guillermo

1977 "El concepto de indio en América: una categoría de la situación colonial", Boletín Bibliográfico de Antropología Americana (1973-1979): 17-32.

Bonfil Batalla, Guillermo

1990 México profundo: una civilización negada. México D. F., Grijalbo.

Canales, Alejandro (y Christian Zlolniski)

2001 "Comunidades transnacionales y migración en la era de la globalización", Notas de población, Año XXVIII, CEPAL: 221-253.

Cuminao, Clorinda

2012 "Construcción de identidades de las vendedoras Kichwas y mestizas y los juegos de poder en el mercado de San Roque", en Eduardo Kingman (coord.), San Roque: indígenas urbanos, seguridad y patrimonio. Quito, Flacso: 79-101.

De la Cadena, Marisol

1992 "Las mujeres son más indias: Etnicidad y género en una comunidad del Cuzco", Revista Isis Internacional, Santiago de Chile Ediciones de las Mujeres, $\mathrm{n}^{\circ} 16$.

Espinosa, Oscar

2009 “¿Cómo se relacionan con lo urbano los indígenas amazónicos peruanos en el siglo XXI?”, Bulletin de l'Institut Français d'Études Andines, oㅜ 38 (1): 47-59.

Figoli, Leonardo (y Dimitri Fazito)

2009 "Redes sociales en una investigación de migración indígena: el caso de Manaus", R. Bras. Est. Pop., v. 26, no 1: 77-95.

Galinier, Jacques (y Antoinette Molinié)

2013 Los neo-indios. Una religión del tercer milenio. Quito, Abdayala.

Hidekazu, Araki

2011 "Movimientos etnicos y multiculturalismo en Ecuador: pueblos indígenas, afrodescendientes, y montubios", Revista electrónica ALAI. Revista Latinoamericana de informacion: 33-57.

Kingman, Eduardo

2002 "Identidad, mestizaje e hibridación: sus usos ambiguos", Proposicones, no 34.

http://www.flacso.org.ec/docs/artidenymestizaje.pdf

Kingman, Eduardo

2003 Discursos y relaciones de poder en la primera mitad del siglo XX. Tarragona, Rovira i Virgili.

Mancero Acosta, Mónica

2013 Nobles y cholos: raza, género y clase en Cuenca. 1995-2005. Quito, FLACSO Sede Ecuador.

Rivadeneira, Lucia

2012 Los Montubios: sujetos étnicos en construcción. Tesis de Maestría. Quito, FLACSO Ecuador.

Sobczyk, Rita (y Rosa María Soriano Miras)

2015 "La dimensión étnica de la identidad: la diáspora comercial de Otavalo", Latinoamérica. Revista de estudios Latinoamericanos, $\mathrm{n}^{\circ}$ 60: 207-237.

Tamagno, Liliana

2003 "Identidades, saberes, memoria histórica y prácticas comunitarias. Indígenas tobas migrantes en la ciudad de La Plata, capital de la provincia de Buenos Aires, Argentina", Campos, no 3: 165-182.

Valcuende del Río, José María

1998 Fronteras, territorios e identificaciones colectivas. Sevilla, Blas Infante. 
Valcuende del Río, José María (y Piedad Vásquez Andrade)

2016 "Orden corporal y representaciones raciales, de clase y género en la ciudad de Cuenca (Ecuador)", Chungara, $n^{\circ} 48$ (2): 301-317.

Varas, José Manuel

2005 Etnificación y etnogénesis del movimiento mapuche urbano organizado en la ciudad de Santiago 1990-2000. Tesis de maestría en Antropología y Desarrollo. Universidad de Chile.

Vásquez, Jorge Daniel

2014 Identidades en transformación. Juventud indígena, migración y experiencia transnaciona en Cañar, Quito, FLACSO Ecuador.

Viveros, Mara

2010 La sexualización de la raza y la racialización de la sexualidad en el contexto latinoamericano actual. Ponencia.pdf (consulta 02/10/2015) 